\title{
Mining tacit knowledge of pilots in flight missions based on visual cognitive graph
}

This paper was downloaded from TechRxiv (https://www.techrxiv.org).

\section{LICENSE}

CC BY 4.0

SUBMISSION DATE / POSTED DATE

02-09-2021 / 10-09-2021

\section{CITATION}

Jin, Dian (2021): Mining tacit knowledge of pilots in flight missions based on visual cognitive graph. TechRxiv. Preprint. https://doi.org/10.36227/techrxiv.16552986.v1

$\mathrm{DOI}$

10.36227/techrxiv.16552986.v1 


\title{
Mining tacit knowledge of pilots in flight missions based on visual cognitive graph
}

\author{
Weiwei Yu, Dian Jin, Zhijun Ren, Xiaokun Zhang
}

\begin{abstract}
As a highly dynamic operating process, flight activity requires a lot of attention from pilots. Thus, it's quite imperative to give research to their visual attention. Traditional research methods mostly based on qualitative analysis, or hypothetical model, and seldom put context information into their model. However, the underlying knowledge (tacit knowledge) hidden in the different performances of pilot's attention allocation is context related, and is hard to express by experts, thus it is difficult to use those traditional methods to construct an interaction system.

In this paper, we mined attention pattern with scene context to achieve the quantitative analysis of tacit knowledge of pilots during flight tasks, and use the method of data mining as well as attribute graph model to construct visual cognitive graph(s). The attribute graph model was adopted to construct visual cognitive graphs which associate the obtained visual information within the flight context. Based on the model, the attention pattern with scene context was mined to achieve the quantitative analysis of tacit knowledge of pilots during flight tasks. Besides, three physical quantities derived from graph theory was introduced to describe the tacit knowledge, which can be used directly to construct an interaction system: first, key information, which shown as central node in the graph we built, reveals the most important information during flight mission within context; second, relevant information, which contains several nodes that was closely connected and strongly impact the central node, reveals the factors affecting the key information; third, bridge information based on betweenness centrality, which can be regard as the important information bridge(s), reveals the process of decision making.

Our work can be directly used to train novice pilots, to guide the interface design, and to construct the adaptive interaction system.
\end{abstract}

Index Terms-Tacit knowledge, Human-machine interactions, Visual cognitive graph

\section{INTRODUCTION}

$\mathbf{F}$ LIGHT activities are highly dynamic operating processes, failure of the pilot to focus attention on specific task can result in catastrophic consequences [1]. Despite the decrease in the events threatening aviation safety over the years, accidents caused by human error have not shown a decreasing rate owing to the upgraded equipment [2]. Endsley revealed that more than $75 \%$ of human error-caused aviation accidents were caused by a pilot failure in perception [3]. Moreover, it was pointed out that about $88 \%$ of all accident causes can be traced

This research has been made possible by "111 Project" (Grant no. B13044) Thanks to the authors of TICC for contributing open source code.

W. Yu, D. Jin and Z. Ren are with the School of Mechanical Engineering, Northwestern Polytechnical University, Xi'an 710072, China (e-mail: yuweiwei@nwpu.edu.cn; d.jin@mail.nwpu.edu.cn; )renzhijun@mail.nwpu.edu.cn

$\mathrm{X}$. Zhang is with the School of Computing and Information Systems, Athabasca University, AB T9S 3A3, Canada (e-mail: xiaokunz@athabascau.ca) back to the situation awareness(SA) [4]. Since a pilot's SA can be assessed by monitoring pilot behavior utilizing observations of pilot eye movement [5], it's quite imperative to study this visual attention.

There are lots of studies focus on human visual attention allocation. Significant differences were found in visual attention between experts and novices [6] leading to different flight performances within the same flight context. For instance, the scanning behavior of several experts and novice pilots was analyzed during simulated approaches and landings tasks. It was found that experts had significantly shorter dwells, more aim points, more total fixations, airspeed fixations, fewer altimeter fixations, and better defined eye-scanning patterns compared to the novices [7]. One experiment revealed significant differences between novices' and experts' eye movement characteristics such as numbers and duration on the areas of interests [8]. In another research, expertise differences were explored in decision-making processes by simultaneously measuring experts' and novice pilots' attention. It was investigated how expertise differences in pilots' attentional strategies were affected by cue properties of diagnosticity and correlation [9]. The better comprehension of the systematic and structured attention in experienced pilots may promote error reduction in training procedures, mitigate operational problems and increase flight safety [10].

Although research above have already discovered lots of regularities of human visual attention, it is difficult to express explicitly the underlying knowledge (tacit knowledge) hidden in the different performances of the pilots' attention allocation. They cannot answer the following questions:

1) What is the imperative knowledge obtained from visual information while operating?

2) How the perceived information relates to each other?

3) What is the related knowledge that influence the pilot's decision?

In fact, the above tacit knowledge as one of the important parts of our cognition is highly personal knowledge [11]. It is difficult to standardize and express to others. It is mainly hidden in personal experience as subjective experience or experience knowledge, which is difficult to describe or express with structural concepts. Thus, it is very difficult for experienced pilots to express and explain their intrinsic knowledge of visual attention regularities. However, discovering the tactic knowledge hidden in the attention behavior of experienced pilots will be a good reference to increment the training capability of operational flight simulators [12]. Hence, a standard can be developed to associate the pilots' level of 
SA with their mode of attention distribution [5]. Moreover, as guidance, the appropriate modality will be selected for presenting in-cockpit information [13].

Therefore, this research aims to analyze the pilot's visual attention allocation, and discover the knowledge hidden in the attention regularities. The remainder of this paper is organized as follows. In the section II, we reviewed some literature related to the visual attention model and tacit knowledge mining. In the section III, the main architecture of the proposed method is presented. In the section IV, high-dimensional flight parameters and interactive operations are used to generate flight task tags. In the section V, the tags obtained in section IV are used as attributes to generate visual cognitive graphs, which is regarded as the attention allocation model. In the section VI, the tacit knowledge is mined from the visual cognitive graphs based on graph theory.

\section{CRITICAL REVIEWS AND RELATED WORK}

In this section, we reviewed the related works from two aspects, one is on the mining method of tacit knowledge which is derived from visual attention, and the other is on the modeling approach of visual attention allocation.

\section{A. Tacit knowledge mined from Visual attention}

Tacit knowledge was first proposed by Michael Polanyi dividing human cognitive forms into two categories. One is explicit knowledge, normally expressed in written words, described as knowledge, diagrams, and mathematical formulas. The other is tacit knowledge such as the knowledge to act in doing something [14]. Tacit knowledge is highly personal knowledge and it is hard to standardize and express to others [11]. A convincing example of tacit knowledge is Polanyi's own description of a student reading an X-ray of a chest [15]. Later, different experiments were organized to discover the existence of tacit knowledge in the field of medical care [15], construction industry [16], [17], medical care [18], [19], art [20], and sport [21], [22]. However, the above research is based on the method of statistical analysis, which is difficult to reveal the intrinsic factors of tacit knowledge.

Eye-movement as the main manifestation of visual attention is a good quantized technique to extract and analyze tacit knowledge. The $x$ and $y$ coordinates were recorded by one research [20] for both eyes of a pottery-making master utilizing an eye-tracking system. The diversity was calculated in attentional focus to reveal the intrinsic tacit knowledge. Analyzing the retrospection of the creative process, a certain consistency was found between the saccade-line and the process. Another experiment [18] was concentrated on the transmission of tacit knowledge in nursing education. By calculating the total gaze time of the nurses and students, significant differences were found in the average ratio of each site's gaze time to the total gaze time, and the average ratio of each site's number of gaze times to the total number of gaze times. Moreover, there was a significant difference between the gaze movement of skilled nurses and nursing students in intravenous injection. Applying eye-tracking to the realm of construction safety practices, researchers analyzed first fixation time, dwell percent, fixation count, run count. They discovered that construction workers' hazard detection and visual search strategies can be significantly enhanced by tacit safety knowledge acquired from work experience and injury exposure [17].

However, tacit knowledge is context specific [11] making it difficult to extract. Five eye movement indexes were recorded and analyzed by some researchers. They discovered that eye movement data were different among three tasks(landing, climbing, cruise flight) [23]. It was also found that the difficulty level of a task can affect the eye movements [7], [24].

\section{B. Attention allocation model concerning context information}

"Context", includes the state of the physical environment (e.g., temperature, noise, etc.), the state of the resources regarding the system surroundings, the state of the system itself and the user's situation (e.g., its location, activity, etc.). The user's situation acquires special importance for achieving the managing user attention challenge [25]. There exist several methods about modeling interactions with context information:

One is based on the Probabilistic inference. Prior inputs and the inference of further outputs are regulated by the strength of probabilistic associations [26] which in visual perception can be manifested in the interdependence of successive fixations [27]. Based on this, A Markov chain was used to analyze the influence of separate guide signs on a driver's gaze transition [28]. However, the actual attentional processing of the individual looking at a scene remains "hidden" in the sense that it cannot be observed directly but can be inferred through the gaze movements [29] [30]. Under this perspective, Hidden Markov Model (HMM) was proposed to model human visual behavior while performing work operations based on visual inspection. For example, the fixation sequences registered within the confines of the defined $\mathrm{AOI}$ (area of interest) were utilized to model the possible hidden visual quality inspection task states. Here context information is used as latent state of a Markov process [31]. [27] use bayesian theories to show an understanding of underlying mechanisms suggesting a typicalities in the evaluation of probabilistic links within the perceptual environment of the affected individual. A dynamic Bayesian network that infers probability distributions over attended objects and spatial locations directly from observed data is introduced in [32] to predict eye fixations. Another research applied Bayesian Hidden Markov Modeling (BHMM) to capture both dynamics and structure of visual exploration of social scenes. BHMM based both on spatial and temporal gaze behavior. Social visual perception was compared between groups using transition and fixation variables for social and non-social Regions of interest(ROIs) [33]. Though a number of papers published recently that applied Probabilistic inference based method in visual attention analysis, most of them focus on classification problem or behavior prediction. The tacit knowledge hidden in these behaviors cannot be recovered.

Artificial Neural Network is also used to improve operation efficiency and safety in human-robot collaboration by considering the context information. [34] introduced DeepFix, which takes global context into account to understanding and predicting the human visual attention mechanism. Visual observation 
of human workers' motion provides informative clues about the specific tasks to be performed, thus can be explored for establishing accurate and reliable context awareness [35]. In traffic safety domain, a Maximum Entropy Deep Inverse Reinforcement Learning (MEDIRL) framework [36] is used to model the visual attention allocation of drivers in imminent rear-end collisions, which takes information includes traffic density, distance to other cars, and brake lights as context information. Similarly, in [37], Capsule Networks is used to model context-driven visual attention by considering different conditions. However, Artificial Neural Network is difficult to explain, thus is hard to find the hidden knowledge or rules.

Since previous studies showed that scan path made by an observer exploring a visual stimulus, can infer both of observer-related and stimuli-related information. Another model is based on graph, including heat maps [38], which can better separate different levels of observation intensity than fixation maps. However, heat maps lose the information about the transitions between fixation, thus saccade plots [39] is introduced. In order to contain temporal information, dynamic graph is used to model human eye movement data, and reveals temporal patterns in the eye movement data [40]. In [41], degree centrality was implied to analyze gaze distribution to explore the influence of bottom-up features. However, those graph model have yielded mixed results. The existing studies mostly quantify eye-movement data only using arbitrary or experimenter defined criteria such as time segments and regions of interests while ignoring how to combine context information and human operation data, thus are hard discover the hidden tacit knowledge.

Although, the related works are focus on either the attention model or the tacit knowledge extraction, there are seldom works on the mining tacit knowledge of attention allocation. Moreover, tacit knowledge is context specific [11] making it difficult to extract. However, the hidden tacit experience is so valuable to novice pilots and meaningful for the operating system design. Therefore, some important tacit knowledge of pilot's attention distribution needs to be revealed, such as,

1) What is the key information that the pilot must obtain within certain context?

2) Are there any group of information that the pilot always has to pay attention, because these information closely connect with the key information(may influence or be influenced by the key information)? For example, the observation of these information is in fact aim to confirm or compare with the key information.

3) What is the bridge information that used as references to connect the previous information and next information for future decision making?

In this paper, flight parameters, operations and eye movement data are used to generate an attribute graph which is called visual cognitive graph, thus context information and pilot information are both contained in one model. Based on the visual cognitive graph, three physical quantities derived from graph theory is introduced to describe the tacit knowledge, first, key information, which shown as central node in the graph we built, reveals the most important information during flight mission within context; second, relevant information, which contains several nodes that was closely connected and strongly impact the central node, reveals the factors affecting the key information; third, bridge information based on betweenness centrality, which can be regard as the important information bridge(s), reveals the process of decision making.

\section{ARCHITECTURE OF MINING TACIT KNOWLEDGE}

Tacit knowledge is context specific, which means that the tacit knowledge should be mined under certain flight situation [11]. Thus, we separated the full mission into several flight stages, and use them as attributes to assign to the eye movement data for the analysis of the context-related tacit knowledge.

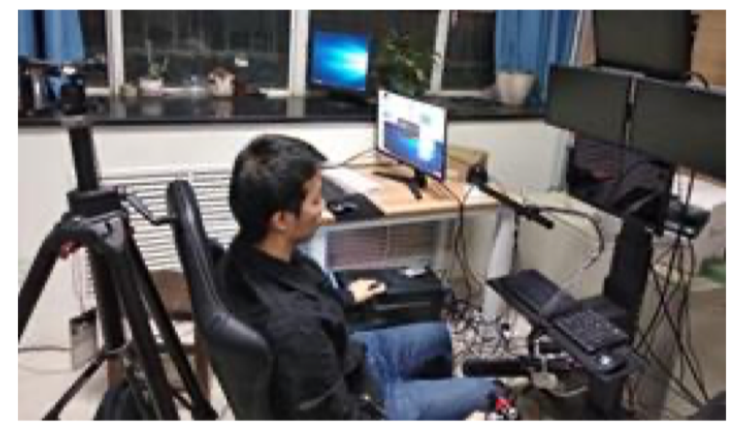

Fig. 1. UAV operation simulator

The operation interface is shown in Figure 1. We take the UAV operation simulator our flight tests platform. The operation mission consists of several flight stages, such as "take off", "climb", "level off", "find target", "lock target", "return". Flight parameters(see section IV-A), pilots' interactive operation and eye movement data(which can be used to extract AOI, see figure 6 and flying scenes 11) are recorded during experiments.

\section{A. Flight context for each stage}

The flight context of important interaction phase for each stage was explained in detail as follows:

a) Take-off: The pilot should check the airfield runway is free of obstacles and make sure the aircraft is facing the takeoff direction. After hearing taking off instruction, the pilot press the button sequentially to make the UAV take-off automatically. At the same time, the subjects should check whether hydraulic pump pressure, brake pressure, oil pump pressure, engine and other conditions are normal. In the take-off stage, the aircraft moves forward in a straight line and accelerates slowly until it is completely off the ground. Then, aircraft further increases the speed and the landing gear retract automatically. The subjects need to cycle check the information of the aircraft's climbing attitude, speed and height.

b) Climbing: When the aircraft reaches the required attitude and the flight is stable, it enters to the climbing stage. At this stage, the speed of the aircraft is basically stable, and the climbing rate is maintained until the altitude gradually 
rises to certain value. According to their own operating habits and experience, the subjects were required to watch the flight parameters such as altitude, speed, pitch angle, main screen display and flight path circularly to monitor the state of the aircraft.

c) Cruising: The default cruise of the UAV is approximate a rectangle, and it flies in the level-flight manner after turning the first turn. In this stage, both of the flight altitude and airspeed should be maintained at certain value. This stage also appears after the subject finishing the task of lock the target, and returning the aircraft back. During this phase, subjects do not need too much operation and mainly need to continuously monitor many flight parameters, such as altitude, speed, roll, pitch and yaw angle, and flight path.

d) Search and lock the target: When arriving the target area, the subject switches the control mode to manual. The subject manually controls the aircraft to hover over the area to search for the target, while both the altitude and speed of the aircraft were required to be maintained within certain deviation, and the rolling angle within certain range. After the target is found in the forward screen display, the UAV control mode is adjusted to command control. At the same time, the subject should adjust the azimuth and elevation ratio of CCD to keep the target in the reconnaissance CCD center and lock the target. While monitoring the target, the subjects should ensure that other flight indicators of the aircraft are normal, the target is accurately displayed on the track chart, and marking related target parameters.

\section{B. Research Methodology}

It's obvious that the performances of subjects are context related, their operation goals in different stages are different even their control requirement is the same. For example, subjects should pay more attention on the main display while keeping the altitude in Cruising stage than that in Climbing stage.

For one simulated flight test, eye movement data of subject are sampled and extracted to form AOI sequence. Then, individual AOI are taken as nodes, and the relevant task tags which extracted from flight parameters, operation data and etc. as attributes are used to construct the attribute graph which we call visual cognitive subgraph.

For several simulated flight tests, several subgraphs can be obtained. By merging them, visual cognitive graph is constructed.

The flow chart is shown as Figure 2. For the convenience of expression, the following definitions is introduced:

Definition 1 (AOI sequence). AOI sequence is a directed chain composed of AOI in chronological order.

Definition 2 (AOI sequence with attributes). Give each AOI in AOI sequence one or several attribute(s), such as flight stage, physiological state, etc..

Definition 3 (Visual cognitive subgraph). In the AOI sequence with attributes, the individual items are taken as nodes to build a graph by measuring their transition relationship.

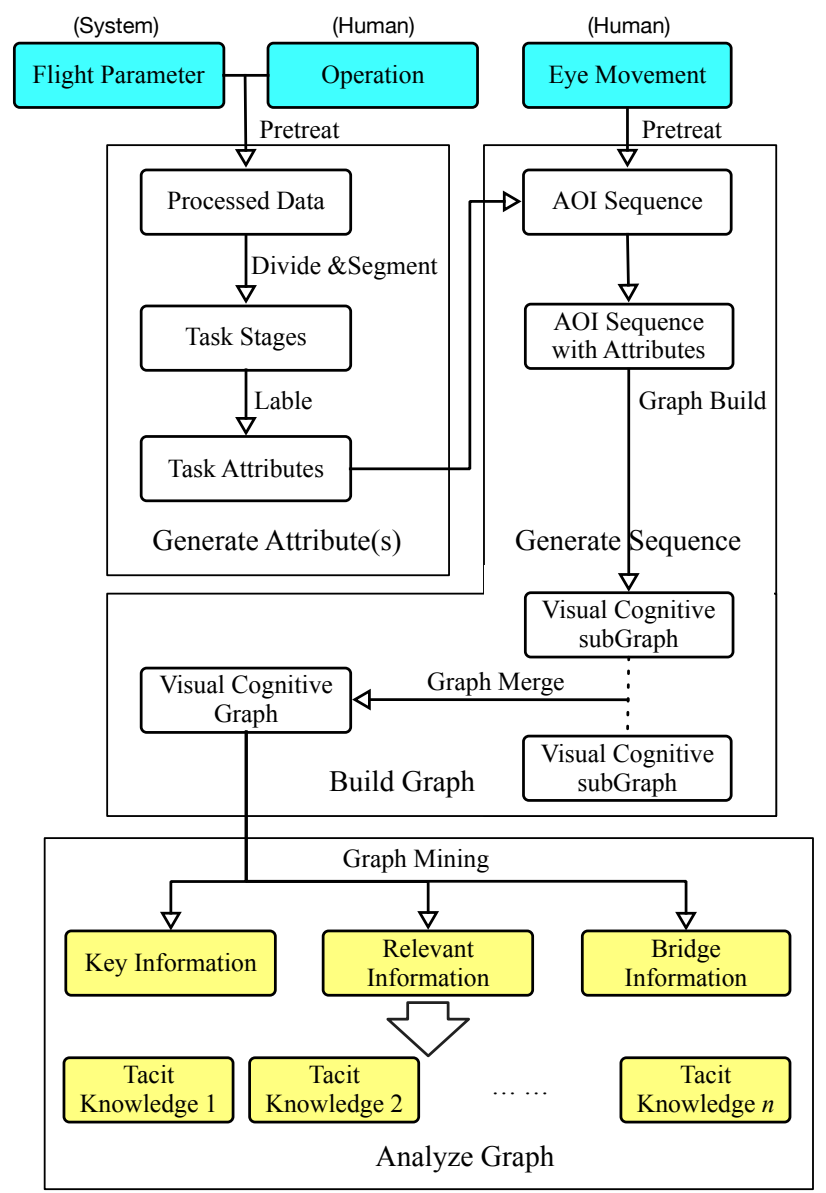

Fig. 2. Architecture of proposed method

Definition 4 (Visual cognitive graph). For several simulated flight tests, several subgraphs can be obtained. By merging them, we can get visual cognitive graph.

\section{GENERATE TASK ATtRibUtes of VisuAl COGNitive GRAPH}

The operation of pilots in a flight is associated with the task scenario. Thus, the attention distribution should be analyzed within the different flight task contexts. However, most of the task division is manually done, requiring repeated playback, which is affected by subjective factors. This is inefficient, and unable to inspect data within correlation to find the hidden task scenario.

This section will put forward a task division method to divide the task scenario and generate the corresponding task tags, which can be used as an attribute of nodes in building the visual cognitive graph.

\section{A. Pretreatment}

The flight tasks were operated while various types of flight parameters were recorded at the same time on the flight simulation platform, such as: "time", "longitude", "latitude", "elevation", "azimuth", "pitch", "roll", "true airspeed", "ground speed", "mach number", "climb rate", "overload", 
"engine status", "EO azimuth", "EO pitch", "EO distance", "pulse rotate speed", "residual fuel".

Some of the flight parameters are continuous, such as "longitude" and "latitude". However others are discrete, such as "engine status". The original flight parameters are characterized by higher dimensions, large amounts, complex types and diverse forms, etc. However, some dimensions of flight parameters overlapped while presenting the flight task scenario, such as "time" and "residual fuel", both revealing the time spent. Thus, after removing outliers of flight data, feature selection should be executed to present the condensed data for subsequent algorithm. In this paper, Mutual Information(MI) was adopted to select features. It is often used to measure the degree of correlation between features and is regarded as the information quantity of another feature contained in one feature. The MI of two discrete random variables $\mathrm{X}$ and $\mathrm{Y}$ is represented as follows:

$$
\mathrm{I}(X, Y)=\mathrm{H}(X)-\mathrm{H}(X \mid Y)
$$

where:

$$
\begin{gathered}
\mathrm{H}(X)=-\sum_{i=1}^{n} \mathrm{p}\left(x_{i}\right) \log \mathrm{p}\left(x_{i}\right) \\
\mathrm{H}(X \mid Y)=\mathrm{H}(X, Y)-\mathrm{H}(Y) \\
\mathrm{H}(X, Y)=-\sum_{i=1}^{n} \sum_{j=1}^{m} \mathrm{p}\left(x_{i}, y_{j}\right) \log \mathrm{p}\left(x_{i}, y_{j}\right)
\end{gathered}
$$

$\mathrm{p}\left(x_{i}\right)$ represents the probability of $x_{i}$ occurring, and $\mathrm{p}\left(x_{i}, y_{j}\right)$ shows the probability of $x_{i}$ and $y_{j}$ occurring.

When one of the two features is a continuous variable, the technique of data discretization is used to divide the data into several intervals.

Then, Relevancy of feature $f_{i}$ and $f_{t}$ is defined as:

$$
\operatorname{Rel}\left(f_{i} \mid f_{t}\right)=\frac{\mathrm{H}\left(f_{i} \mid f_{t}\right)}{\mathrm{H}\left(f_{i}\right)} \operatorname{Rel}\left(f_{i}\right)
$$

where:

$$
\operatorname{Rel}\left(f_{i}\right)=\frac{1}{n} \sum_{j=1}^{n} \mathrm{I}\left(f_{i}, f_{j}\right)
$$

and $\mathrm{H}\left(f_{i} \mid f_{t}\right)$ represent the uncertainty of the feature $f_{i}$ under given $f_{t}$.

Define Redundancy:

$$
\operatorname{Red}\left(f_{i} ; f_{t}\right)=\operatorname{Rel}\left(f_{t}\right)-\operatorname{Rel}\left(f_{t} \mid f_{i}\right)
$$

Fig. 3 shows the heat map of the redundancy matrix, where the number in each cell reveals the degree of redundancy between two data. Taking "time" \& "residual fuel" as an example, it is found that they own a large redundancy, representing the same trend in the mission.

When choosing the $m^{\text {th }}$ feature, the correlation of candidate features and their redundancy are comprehensively considered for selected features. Moreover, unsupervised minimum redundancy - maximum relevant index(UmRMR) of each feature is obtained. The UmRMR of feature $f_{i}$ is:

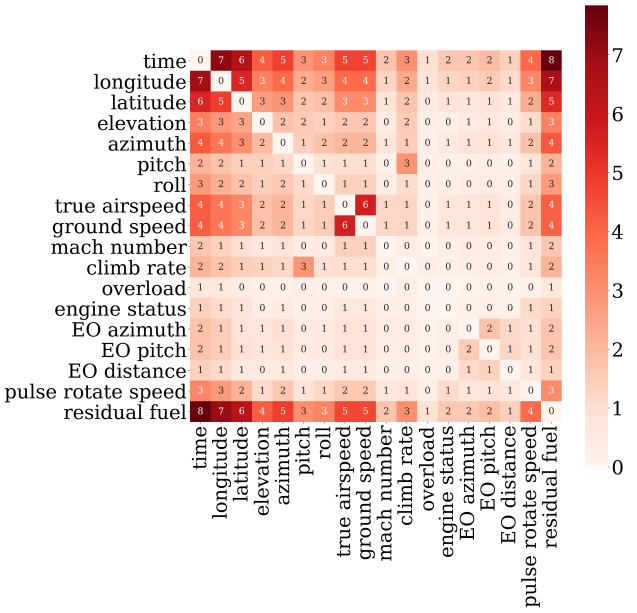

Fig. 3. Redundancy heat map

$$
\operatorname{UmRMR}\left(f_{i}\right)=\operatorname{Rel}\left(f_{i}\right)-\max _{g \in \mathrm{S}_{m-1}}\left\{\operatorname{Red}\left(f_{i} ; g\right)\right\}
$$

where $\mathbf{S}_{m-1}$ is the set of features except $f_{i}$.

Thus we can select several important features among all those features.

Furthermore, the magnitude of flight parameters is quite different from each other, imposing a negative impact on data analysis. Thus, the data should be normalized.

$\boldsymbol{x}_{i}$ represents the original data vector. After standardization, the formula for calculating $\boldsymbol{y}_{i}$ is:

$$
\boldsymbol{y}_{i}=\frac{\boldsymbol{x}_{i}}{\max \left(\operatorname{abs}\left(\boldsymbol{x}_{i}\right)\right)}
$$

The raw data include Flight Parameters\&Operation data and Eye movement data. Flight Parameters\&Operation data was recorded by 79 indexes and has around 3500 items for each experiment. Eye movement data have 3 indexes: the coordination of fixation $(x, y)$, start time and end time, with around 1000 items for each experiment.

After the pretreatment, for Flight Parameters\&Operation data, 18 indexes were selected that shown significant changes during the experiments, then 10 indexes were selected by calculating the Redundancy. The dimension is reduced to $10 \times 3500$ approximately for each experiment.

\section{B. Flight task scenario division}

For task-oriented scenario, such as UAV operating, flight task heavily affect people's visual attention distribution. In this section, the tasks scenario would be segmented and clustered with flight scene context. The differences in the characteristics of flight task are mainly related to the task content which will be reflected by the flight parameters. The flight parameters data are from sensors of the airplane, sensors of load and flight mode. According to the task timeline, these flight parameters will form higher dimensional time series. However, grouping this time series based on the task content is challenging, as it requires simultaneously segmenting and clustering the time sequence. 


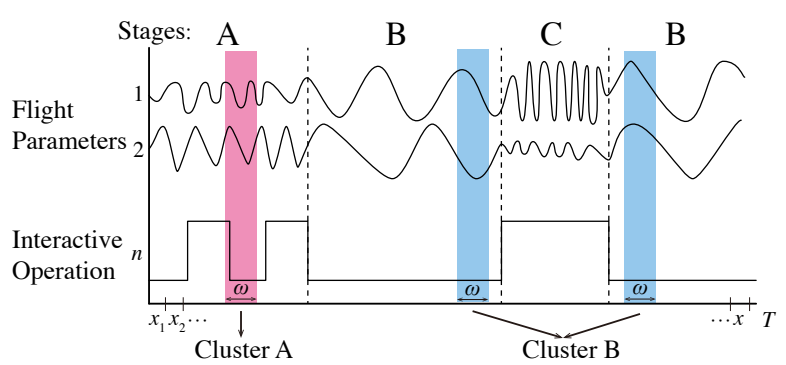

Fig. 4. TICC method segments a time series into a sequence of states, or clusters (i.e., A, B, or C). Each cluster is characterized by a correlation network, or MRF, determined over a short window of size $\omega$. This MRF governs the (time-invariant) partial correlation structure of any window within a related segment. Here, TICC learns both the cluster MRFs and the time series segmentation [42]. Here, $x_{n}$ represents high-dimensional vectors at time point $n, \omega$ represents time window.

The flight parameters which represent simulator system status are continuous, and the operation status data which represent human decision are discrete. These two types of temporal data consist of the context information. In order to discover repeated patterns in this high-dimensional temporal data, we suggested to apply Toeplitz Inverse Covariance-based Clustering (TICC) algorithm [42] to segment the time series of flight parameters. The TICC algorithm segments a time series into a sequence of clusters, characterized by the correlation network, Markov Random Field (MRF). Then, TICC alternates between assigning points to clusters in a temporally consistent way to discover these clusters, which accomplishes through dynamic programming and the Alternating Direction Method of Multipliers (ADMM). Regarding MRF characterizing the interdependencies and correlations between various observations in a typical subsequence of that cluster, the TICC method can segment flight parameters based on the flight scene context.

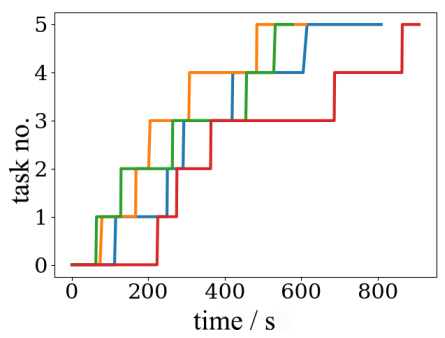

Fig. 5. The results of several experimental flight missions were divided. Different colors represent different experiments

In Fig.5, each of 4 experimental flight mission was divided into 6 stages. They were defined as "take off","climb", "cruise", "find target", "lock target", "return" based on prior knowledge.

\section{Visual COGNITIVE GRAPH}

Eye-fixation as the main manifestation of visual attention, is a good quantized technique for presenting the rules of attention allocation. However, the sequence of AOI with attributes is an unidirectional chain. To extract the hidden rules of pilots' attention and tacit knowledge, it is essential to build a graph integrating the information. We call this attribute graph a visual cognitive graph.

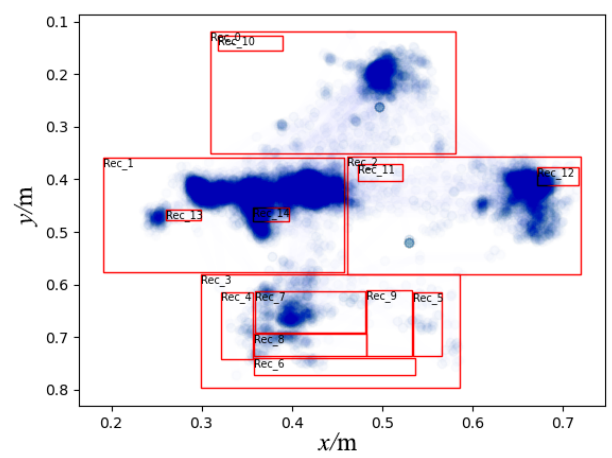

Fig. 6. AOI heat map for one flight test

Each gaze point is assigned into an AOI, as Fig.6, the UAV operating interface is represented by the big rectangular box, while the AOI was defined based on the function region.

The original eye movement data are a sequence of points. To get fixation points sequence, all the null values and outliers were removed, and the fixation points were detected. Then, the data were compressed by merging continuous and repeating AOI to get the AOI sequence.

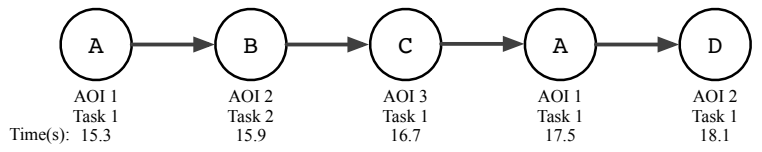

Fig. 7. Eye movement pattern sequence

\section{A. Graph building}

After the pretreatment of the eye movement data, each AOI is labeled with its attributes(e.g., "stage 5"), and it is put into the graph as a node. A directed edge is added when there is a transition relationship between nodes, to construct the sequence of these nodes. For example, as shown in Fig.7, each node is connected by a directed edge, and each node has an in arrow and an out arrow. However, there are numerous duplicate nodes in the graph. To construct a visual cognitive graph, these repeated nodes are combined to form a directed weighted graph $G$ by introducing the concept of transition number matrix.

To explain the building procedure of visual cognitive graph, transition matrix was defined in definition as follows:

Definition 5 (Transition matrix). Considering a single node sequence $L$, there are $k$ non-repeating nodes. Then, a $k$-order matrix is defined, when there is a connection from node $m$ to node $n$ in $L, 1$ is added to the row $m$ column $n$ of the matrix. Since there may be multiple connections between node $m$ and node $n$ in $L$, each term of the matrix is a non-negative integer. The matrix is defined as the transition matrix $C$ of the graph $G$ obtained followed by merging the eye movement node in sequence $L$. 


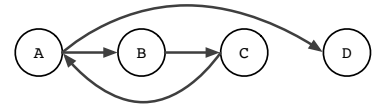

Fig. 8. Transition graph

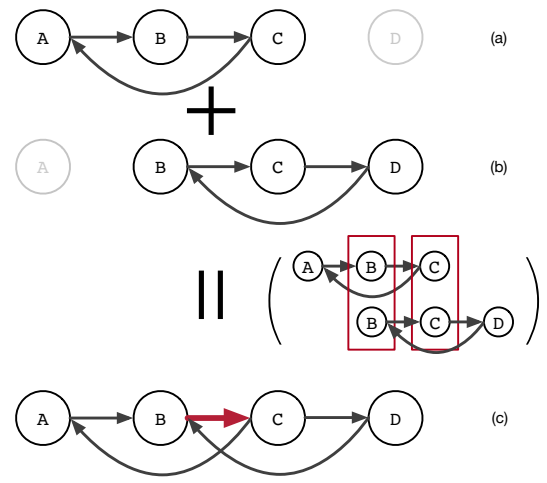

Fig. 9. (a) is an example of visual cognitive subgraph $G_{1}$, (b) is an example of visual cognitive subgraph $G_{2}$, (c) is an example of visual cognitive graph (merged graph) $G$

The same AOI with different flight stage attributes is regarded as different. They should appear as different nodes, as in Fig.8, where each node is unique. The directed edge represents the transition relationship between nodes, and directed edge weight can represent the number of transitions or transition probability or their product. The adjacent matrix of the graph is the transition matrix.

\section{B. Visual cognitive graph merging}

After operating numerous simulated flight tests based on the recorded flight parameters and the pilot's eye movement data, multiple graphs can be obtained, known as visual cognitive subgraphs. To synthesize these larger data sets and discover more general and accurate rules, we should merge these isolated subgraphs.

1) Problem statement: Considering two graphs $G_{1}$ and $G_{2}$, there may exist repeated nodes in $G_{1}$ and $G_{2}$ with the same label. The graph $G$ is found containing all the nodes in the graph $G_{1}$ and $G_{2}$ without repeat. Moreover, for the edge, when the starting node and the target node of an edge are both repeated nodes, which means the edge is repeated(eg. $e_{1}$ in the $G_{1}$ with weight of $w_{1}$ and $e_{2}$ in the $G_{2}$ with weight of $\left.w_{2}\right)$. Then in graph $G$, the weight of the edge is $w_{1}+w_{2}$. When there is a non-repeating node between the starting node and the target node of an edge, which means the edge is not a repeating edge(eg. $e_{3}$ in the $G_{1}$ with weight of $w_{3}$. Then the weight of the edge is $w_{3}$ in graph $G$ (Fig.9).

2) Algorithm implementation:

Input: two matrices $\boldsymbol{B}_{n \times n}$ and $\boldsymbol{I}_{m \times m}$ obtained from $G_{1}$ and $G_{2}$, corresponding node label $\left\{L_{\boldsymbol{B}}\right\}$ and label $\left\{L_{\boldsymbol{I}}\right\}$;

Output: merged matrix $\boldsymbol{M}$, corresponding label $\left\{L_{M}\right\}$.

1: $L_{\boldsymbol{B}}^{\prime}=L_{\boldsymbol{B}}, L_{\boldsymbol{I}}^{\prime}=L_{\boldsymbol{I}}, l_{\boldsymbol{B}}=\left[1, \cdots, \mathrm{N}\left(L_{\boldsymbol{B}}\right)\right], l_{\boldsymbol{I}}=\left[1, \cdots, \mathrm{N}\left(L_{\boldsymbol{I}}\right)\right]$, $c=0$

2: for $i=0 ; i<\mathrm{N}\left(L_{B}\right) ; i++$ do

3: $\quad$ for $j=0 ; j<\mathrm{N}\left(L_{I}\right) ; j++$ do

4: $\quad$ if $L_{B}[i]==L_{B}^{\prime}$ then

5: $\quad c=c+1$

6: $\quad l_{B} \cdot \operatorname{remove}(i), l_{I} \cdot \operatorname{remove}(j)$

7: $\quad L_{B}^{\prime} \cdot \operatorname{remove}\left(L_{B}[i]\right), L_{I}^{\prime} \cdot \operatorname{remove}\left(L_{I}[j]\right)$

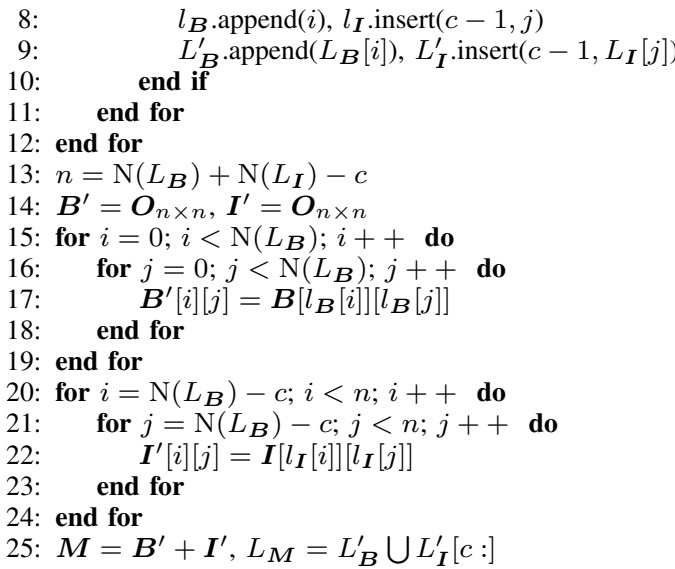

Thus, the visual cognitive graph comprising the information of multiple flight tests can be obtained. Furthermore, the obtained data sets from the new flight trails can be simply synthesized with the visual cognitive graph, to mine further general rules.

\section{TACIT KNOWLEDGE Mining}

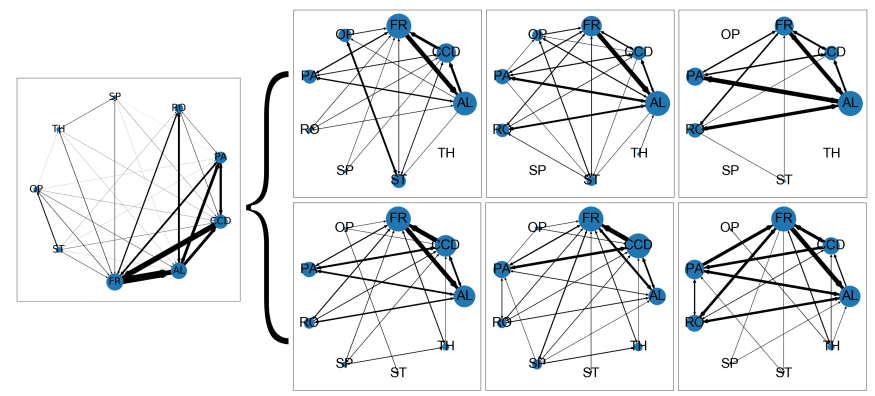

(a)

(b)

Fig. 10. (a)Visual graph without context information(subtask here), (b)Visual cognitive graph with context information can reveal much more patterns. The size of nodes represents their frequency of occurrence. The thickness of lines represents their frequency of transition

Our proposed attention allocation model does not lose context information. To build the visual cognitive graph for mining tacit knowledge, eye-movement data were used as nodes, combined with attributes which represent context information generated by operation data and flight parameters .

Each AOI is assigned a name. "OP" represents "Operation interface", "ST" is "State interface", "CCD" denotes "CCD view", "FR" shows "Front view", "TH" is "Throttle speed", "RO" represents "Roll angle", "SP" denotes "Flight speed", "AL" represents "Flight altitude" and "PA" shows "Flight path".

The visual graph based on the fixation transition for one flight task experiment was shown in In Fig.10(a). In the figure, the size of nodes represents the fixation frequency in one AOI, while the thickness of lines represents the transition frequency between different AOI. However, the context information was ignored in the graph, the detailed information related to flight stages can not be extracted. For the same flight task experiments, the visual cognitive graph based on our proposed method was shown in Fig.10(b). Since the model associated the flight context information with the attributes of the graph, 
the visual graph can reveal the details of attention allocation pattern for every flight stage.

\section{A. Key information mining based on central node}

In a large visual cognitive graph $G$, when there is a higher transition probability from node $v_{i}$ to node $v_{j}$ is high, their distance is defined as short. For the small or non-existent transition probability between $v_{i}$ and $v_{j}$, then the distance is defined as far. For instance, the altitude information is always the most important in the flight stage "climb" and the CCD view/ front view is always important in the stage "find target". Here, the random walk distance is utilized to measure the closeness between nodes.

Definition 6 (Random walk distance). Let $\boldsymbol{P}$ as the transition probability matrix of $n \times n$ of graph $G$, and $c \in(0,1)$ as the restart probability. Then the random walk distance $\mathrm{d}\left(v_{i}, v_{j}\right)$ from $v_{i}$ to $v_{j}$ is defined as:

$$
\mathrm{d}_{k}\left(v_{i}, v_{j}\right)=(1-c) \boldsymbol{P} \mathrm{d}_{k-1}\left(v_{i}, v_{j}\right)+c \boldsymbol{e}
$$

The matrix form of the random walk distance is:

$$
\boldsymbol{R}_{k}=(1-c) \boldsymbol{P} \boldsymbol{R}_{k-1}+c \boldsymbol{E}
$$

Where $e$ is the starting node of matrix, $\boldsymbol{E}$ is the identity matrix. Based on Equation 10, the random walk distance matrix will converge to:

$$
\boldsymbol{R}_{k}=c[\boldsymbol{E}-(1-c) \boldsymbol{P}]^{-1}
$$

Then, the structural intimacy (random walk distance) between two nodes $v_{i}$ and $v_{j}$ is:

$$
\mathrm{d}\left(v_{i}, v_{j}\right)=\boldsymbol{R}_{k}(i, j)
$$

Definition 7 (Unified Neighborhood Random Walk Distance). Let $\boldsymbol{P}_{A}$ as the transition probability matrix of attribute augmented graph $G_{a}$. Considering $c \in(0,1)$ as restart probability, the random walk distance $\boldsymbol{R}$ in $G_{a}$ is determined as:

$$
\boldsymbol{R}=c\left[\boldsymbol{E}-(1-c) \boldsymbol{P}_{A}\right]^{-1}
$$

Where $\boldsymbol{E}$ represents the identity matrix.

Since $G$ is a directed graph, we defined the random walk distance as the average of the random walk distance from node $v_{i}$ to node $v_{j}$. Thus the random walk distance from node $v_{j}$ to node $v_{i}$ is:

$$
\boldsymbol{R}_{A}=\frac{\left(\boldsymbol{R}+\boldsymbol{R}^{T}\right)}{2}
$$

$\boldsymbol{R}_{A}$ represents a unified neighborhood random walk distance matrix, which is a symmetric matrix. This is called unified random walk distance matrix.

By obtaining $\boldsymbol{R}$, we can utilize $\boldsymbol{r}=\sum_{i=1}^{n} \boldsymbol{R}_{A}(i, j)$ to determined the importance of each node, where $\boldsymbol{r}$ is the $n$ dimensional vector, and $\boldsymbol{R}_{A}$ denotes $n \times n$ matrix.

The node with the largest importance is known as central node(eg. "AL" in Fig.11(b)). It represents highly dynamic vital information, as the leading information impacting other information or seriously impacted by other information. It is indicated that altitude is the main information under this task,

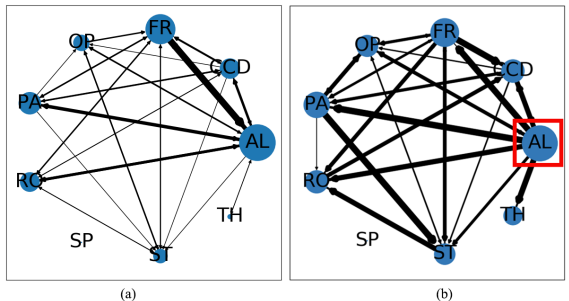

Fig. 11. (a)Visual cognitive graph under flight stage "climb", several experiments done by one subject;(b)Nodes based on random walk distance under flight stage "climb"

representing the altitude as a key information during flight stage "climb". This can be considered as the main part of tacit knowledge.

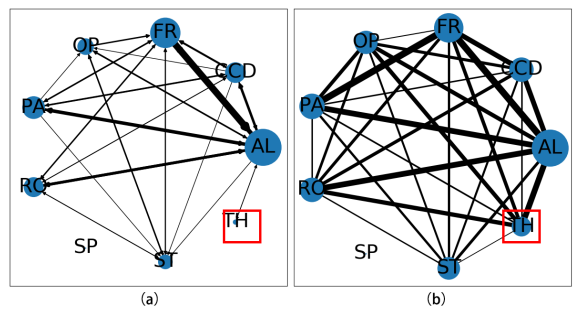

Fig. 12. Under flight stage "climb": (a) Results based on statistics analysis, (b) Results based on random walk algorithm. (The two graphs were modeled based on visual cognitive graph. The size of nodes represents their "importance". The thickness of lines represents the closeness of nodes)

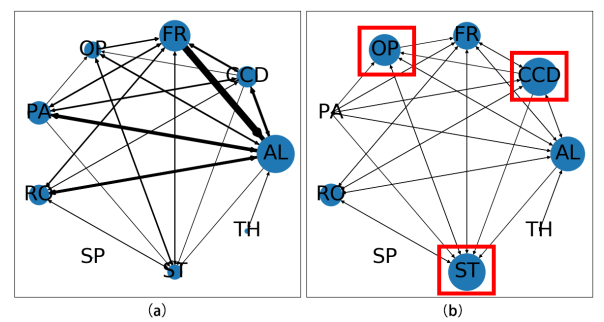

Fig. 13. Under flight stage "climb": (a) Results based on statistics analysis, (b) Results based on betweenness centrality(The thickness of lines means nothing here).

\section{B. Relevant information mining based on central node}

Nodes closely connected to the central node represent the relevant information. They show a series of important information relevant to the key information. The traditional analysis technique is based on statistics. It can only explore the linear relationship between AOI. Our graph theory based method can explore the association between AOI which are not connected in time series. The differences between these two methods are compared in Fig.12. If we only calculate the frequency of fixations in one AOI based on statistics analysis, the importance of "TH"(throttle speed) may be ignored since it less appears. However, this information is imperative to flight stage "climb". It only has a weak connection with the key information "AL", utilizing random walk algorithm, a strong connection can be found, which can be regarded as the manifestation of tacit knowledge. 


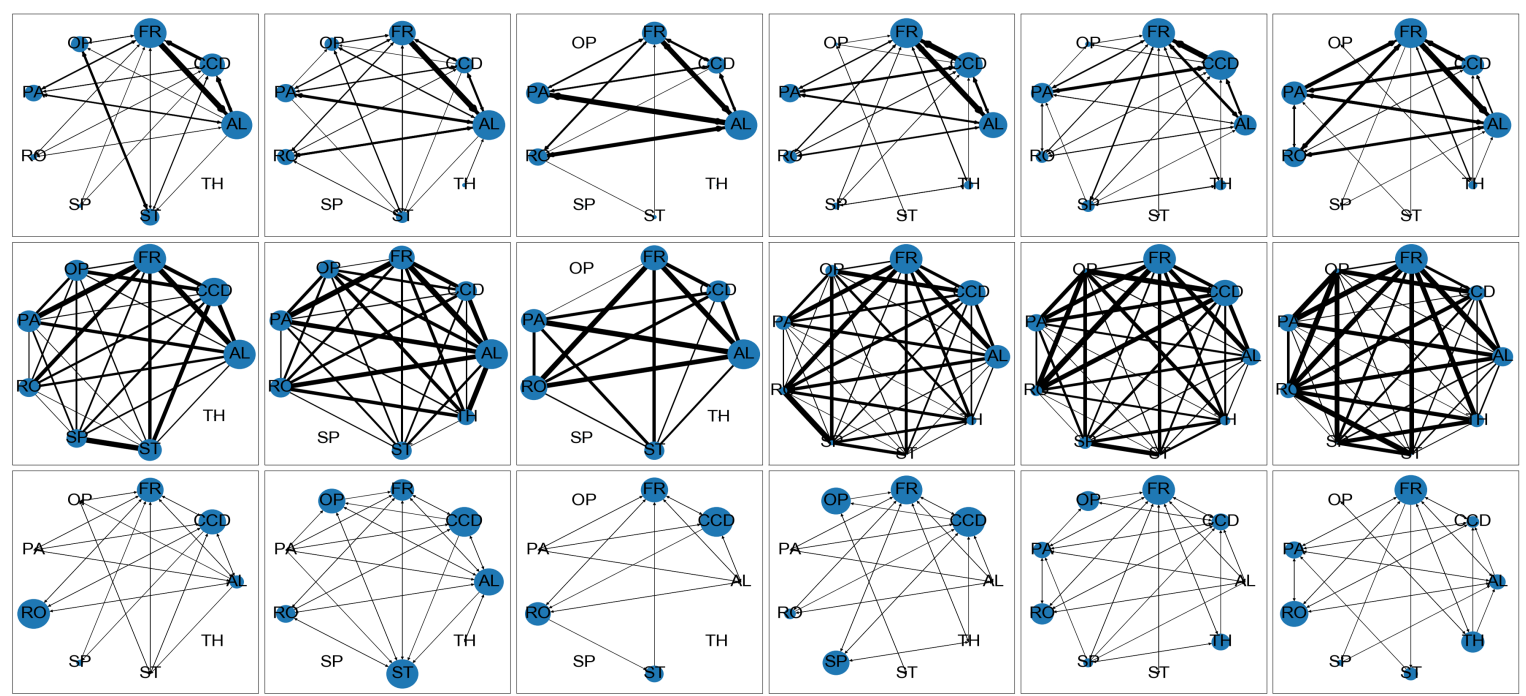

Fig. 14. Six column represent six stages/labels("take off", "climb", "cruise", "find target", "lock target", "return"), the first row is built by statistics analysis, the second row represents the results based on random walk distance and the third row reveals the results based on betweenness centrality

\section{Bridge information mining based on betweenness centrality}

Betweenness centrality [43] of a node $v$ is the sum of the fraction of all-pairs shortest paths that pass through $v$.

$$
c_{B}(v)=\sum_{s, t \in V} \frac{\sigma(s, t \mid v)}{\sigma(s, t)}
$$

where $V$ represents the set of nodes, $\sigma(s, t)$ shows the number of shortest $(s, t)$-paths, and $\sigma(s, t \mid v)$ is the number of paths passing through some node $v$ other than $s, t$. If $s=t, \sigma(s, t)=$ 1 , and if $v \in s, t, \sigma(s, t \mid v)=0$.

We used betweenness centrality to evaluate the importance of an AOI as a bridge connecting other AOIs, as Fig.13. In this trail, the "ST", "CCD", "OP" nodes are much larger. Which indicates that pilot use these information(the state of plane, the operation interface, the CCD view) as references to connect the previous information and next information for future decision making. It can be determined as an indispensable process in most process. For the further application, these several key "bridge" nodes can be utilized for evaluating the status of a pilot since he/she may reduce processing of intermediate processes in a state of fatigue or as a guide to guide the interface design. In Fig.14, visual cognitive graphs of six flight stages("take off", "climb", "cruise", "find target", "lock target", "return") are presented. The first row is built by statistics analysis, the second row represents the results based on random walk distance and the third row reveals the results based on betweenness centrality. Take stage "cruise"(column 3) as an example, we can obtain that "AL" is the central node(column 3, row 1), which means that pilot gave most attention on "altitude". Then in the second part(column 3, row 2), it is shown that "FR", "PA", "RO", "ST" are relevant information, where, "ST" become large comparing to "ST" in the first part(column 3, row 1). That means flight status is an important information to process with the key information "altitude". In the third part(column 3, row 3), "CCD" is the bridge information, which means pilot always use the CCD view as a reference for decision making.

\section{CONCLUSION AND FUTURE WORK}

In order to mine and achieve the quantitative analysis of "tacit knowledge", this article based on the perspective of pilots and used their eye movement data with scene context to construct our model: first, TICC is used to achieve the task division under the flight parameters of high-dimensional and large data volume; then, the divided data is used as task tag along with AOI data to build visual cognitive subgraph; third, under several experiments, we use the method of graph merge to build visual cognitive graph.

We try to reveal their tacit knowledge from three aspect: first, central node, which is the main node being processed as results information, reveals the key information during flight mission under some context; second, relevant information, which contains several nodes that closely connect and strongly impact to the central nodes, reveals the factors affecting the key information; third, bridge information based on betweenness centrality, which can be regard as the important information bridge(s), reveals the process of connecting information. Our work can be directly used to train novice pilots, to guide the interface design, to construct the adaptive interaction system.

Our visual cognitive graph is an attribute graph, which means that it can contain multiple attributes. In this paper, we only assign one attribute "task" to each AOI node and we plan to add more attributes such as workload, operation level, mental status etc. into this graph in the future. Besides, an adaptive interaction system based on this study will be constructed in our future work.

\section{REFERENCES}

[1] J. W. Crandall, M. L. Cummings, M. Della Penna, and P. M. De Jong, "Computing the effects of operator attention allocation in human control of multiple robots," IEEE Transactions on Systems, Man, and 
Cybernetics Part A:Systems and Humans, vol. 41, no. 3, pp. 385-397, 2011.

[2] C.-K. Hsu, S.-C. Lin, and W.-C. Li, "Visual movement and mentalworkload for pilot performance assessment," in International Conference on Engineering Psychology and Cognitive Ergonomics. Springer, 2015, pp. 356-364.

[3] D. G. Jones and M. R. Endsley, "Sources of situation awareness errors in aviation," Aviation Space \& Environmental Medicine, vol. 67, no. 6, pp. 507-512, 1996.

[4] C. M. Muehlethaler and C. P. Knecht, "Situation Awareness Training for General Aviation Pilots using Eye Tracking," IFACPapersOnLine, vol. 49, no. 19, pp. 66-71, 2016. [Online]. Available: http://dx.doi.org/10.1016/j.ifacol.2016.10.463

[5] K. Kilingaru, J. W. Tweedale, S. Thatcher, and L. C. Jain, "Monitoring pilot 'Situation Awareness'," Journal of Intelligent and Fuzzy Systems, vol. 24, no. 3, pp. 457-466, 2013. [Online]. Available: http://dx.doi.org/10.3233/IFS-2012-0566

[6] W.-C. Li, F.-C. Chiu, Y.-S. Kuo, and K.-J. Wu, "The investigation of visual attention and workload by experts and novices in the cockpit," in Lecture Notes in Computer Science (including subseries Lecture Notes in Artificial Intelligence and Lecture Notes in Bioinformatics), vol. 8020 LNAI, no. PART 2, Las Vegas, NV, United states, 2013, pp. 167-176. [Online]. Available: http://dx.doi.org/10.1007/978-3-642-39354-9-19

[7] M. Andrzejewska and A. Stolińska, "Comparing the difficulty of tasks using eye tracking combined with subjective and behavioural criteria," Journal of Eye Movement Research, vol. 9, no. 3, pp. 1-16, 2016.

[8] S. Naeeri and Z. Kang, "Exploring the relationship between pilot's performance and fatigue when interacting with cockpit interfaces," IISE Annual Conference and Expo 2018, no. May 2018, pp. 1498-1503, 2018.

[9] A. T. Schriver, D. G. Morrow, C. D. Wickens, and D. A. Talleur, "Expertise differences in attentional strategies related to pilot decision making," Human Factors, vol. 50, no. 6, pp. 864-878, 2008.

[10] J. M. Diaz, C. Bil, A. G. Dyer, and J. E. Garcia, "Visual scan patterns of expert and cadet pilots in VFR landing," 17th AIAA Aviation Technology, Integration, and Operations Conference, 2017, no. June, pp. 1-11, 2017.

[11] V. Ambrosini and C. Bowman, "Tacit knowledge: Some suggestions for operationalization," Journal of Management Studies, vol. 38, no. 6, pp. 811-829, 2001.

[12] P. A. Wetzel, G. M. Anderson, and B. A. Barelka, "Instructor use of eye position based feedback for pilot training," Proceedings of the Human Factors and Ergonomics Society, vol. 2, pp. 1388-1392, 1998.

[13] C. D. Wickens, J. Goh, J. Helleberg, W. J. Horrey, and D. A. Talleur, "Attentional Models of Multitask Pilot Performance Using Advanced Display Technology," Human Factors, vol. 45, no. 3, pp. 360-380, 2003.

[14] M. Polanyi, "Personal knowledge, personal knowledge," 1958.

[15] P. J. Heiberg Engel, "Tacit knowledge and visual expertise in medical diagnostic reasoning: Implications for medical education," Medical Teacher, vol. 30, no. 7, 2008 .

[16] R. J. Dzeng, C. T. Lin, and Y. C. Fang, "Using eye-tracker to compare search patterns between experienced and novice workers for site hazard identification," Safety Science, vol. 82, pp. 56-67, 2016. [Online] Available: http://dx.doi.org/10.1016/j.ssci.2015.08.008

[17] S. Hasanzadeh, B. Esmaeili, and M. D. Dodd, "Measuring the impacts of safety knowledge on construction workers' attentional allocation and hazard detection using remote eye-tracking technology," Journal of Management in Engineering, vol. 33, no. 5, 2017.

[18] Y. Maekawa, Y. Majima, and M. Soga, "Quantifying eye tracking between skilled nurses and nursing students in intravenous injection," Studies in Health Technology and Informatics, vol. 225, pp. 525-529, 2016.

[19] L. Meerabeau, "Tacit nursing knowledge: an untapped resource or a methodological headache?" Journal of Advanced Nursing, vol. 17, no. 1, pp. 108-112, 1992.

[20] J. Nakamura and S. Nagayoshi, "The pottery skills and tacit knowledge of a maser: An analysis using eye-tracking data," Procedia Computer Science, vol. 159, pp. 1680-1687, 2019. [Online]. Available: https://doi.org/10.1016/j.procs.2019.09.338

[21] B. Abernethy, R. J. Neal, and P. Koning, "Visual-perceptual and cognitive differences between expert, intermediate, and novice snooker players," Applied Cognitive Psychology, vol. 8, no. 3, pp. 185-211, 1994.

[22] B. Abernethy, J. Baker, and J. Côté, "Transfer of pattern recall skills may contribute to the development of sport expertise," Applied Cognitive Psychology, vol. 19, no. 6, pp. 705-718, 2005.

[23] C. Yang, Z. Liu, Q. Zhou, F. Xie, and S. Zhou, "Analysis on eye movement indexes based on simulated flight task," in Lecture Notes in Computer Science (including subseries Lecture Notes in Artificial Intelligence and Lecture Notes in Bioinformatics), vol. 8532 LNAI,
Heraklion, Crete, Greece, 2014, pp. 419-427. [Online]. Available: http://dx.doi.org/10.1007/978-3-319-07515-0_42

[24] R. Dewhurst, T. Foulsham, H. Jarodzka, R. Johansson, K. Holmqvist, and M. Nyström, "How task demands influence scanpath similarity in a sequential number-search task," Vision Research, vol. 149, no. August 2017, pp. 9-23, 2018. [Online]. Available: https://doi.org/10.1016/j.visres.2018.05.006

[25] M. Gil, M. Albert, J. Fons, and V. Pelechano, "Designing human-inthe-loop autonomous Cyber-Physical Systems," International Journal of Human Computer Studies, vol. 130, no. June 2018, pp. 21-39, 2019. [Online]. Available: https://doi.org/10.1016/j.ijhcs.2019.04.006

[26] G. Boccignone, "Advanced statistical methods for eye movement analysis and modeling: a gentle introduction," arXiv, 2015.

[27] M. Otten, A. K. Seth, and Y. Pinto, "A social bayesian brain: How social knowledge can shape visual perception," Brain and cognition, vol. 112, pp. 69-77, 2017.

[28] T. Shang, H. Lu, P. Wu, and X. Lu, "Method of Setting Exit Advance Guide Signs in Highway Tunnels Based on the Driver's Eye Movement with Markov Chains," IEEE Access, vol. PP, no. 99, p. 1, 2021.

[29] M. Hayashi, "Hidden markov models to identify pilot instrument scanning and attention patterns," in SMC'03 Conference Proceedings. 2003 IEEE International Conference on Systems, Man and Cybernetics. Conference Theme-System Security and Assurance (Cat. No. 03CH37483), vol. 3. IEEE, 2003, pp. 2889-2896.

[30] J. Grobelny and R. Michalski, "Applying hidden Markov models to visual activity analysis for simple digital control panel operations," WORking papers in Management Science (WORMS), 2017.

[31] B. Ulutas, F. Ozkan, and R. Michalski, "Application of hidden Markov models to eye tracking data analysis of visual quality inspection operations," WORking papers in Management Science (WORMS), 2020.

[32] A. Borji, D. N. Sihite, and L. Itti, "What/where to look next? Modeling top-down visual attention in complex interactive environments," IEEE Transactions on Systems, Man, and Cybernetics: Systems, vol. 44, no. 5, pp. 523-538, 2014.

[33] C. Ioannou, D. Seernani, M. E. Stefanou, M. Biscaldi-Schaefer, L. T. Van Elst, C. Fleischhaker, G. Boccignone, and C. Klein, "Social visual perception under the eye of Bayesian theories in autism spectrum disorder using advanced modeling of spatial and temporal parameters," Frontiers in psychiatry, vol. 11, 2020.

[34] S. S. Kruthiventi, K. Ayush, and R. V. Babu, "DeepFix: A Fully Convolutional Neural Network for Predicting Human Eye Fixations," IEEE Transactions on Image Processing, vol. 26, no. 9, pp. 4446-4456, 2017.

[35] P. Wang, H. Liu, L. Wang, and R. X. Gao, "Deep learning-based human motion recognition for predictive context-aware human-robot collaboration," CIRP Annals, vol. 67, no. 1, pp. 17-20, 2018. [Online]. Available: https://doi.org/10.1016/j.cirp.2018.04.066

[36] M. Wulfmeier, P. Ondruska, and I. Posner, "Maximum entropy deep inverse reinforcement learning," arXiv preprint arXiv:1507.04888, 2015.

[37] J. Martinez-Cebrian, M. A. Fernandez-Torres, and F. Diaz-De-Maria, "Interpretable Global-Local Dynamics for the Prediction of Eye Fixations in Autonomous Driving Scenarios," IEEE Access, vol. 8, pp. $217068-217085,2020$.

[38] O. Špakov and D. Miniotas, "Visualization of eye gaze data using heat maps," Elektronika ir elektrotechnika, vol. 74, no. 2, pp. 55-58, 2007.

[39] M. Burch, H. Schmauder, M. Raschke, and D. Weiskopf, "Saccade Plots," Eye Tracking Research and Applications Symposium (ETRA), pp. 307-310, 2014.

[40] M. Burch, F. Beck, M. Raschke, T. Blascheck, and D. Weiskopf, "A dynamic graph visualization perspective on eye movement data," Eye Tracking Research and Applications Symposium (ETRA), vol. 1, pp. 151158, 2014.

[41] P. Yazdan-Shahmorad, N. Sammaknejad, and F. Bakouie, "Graph-Based Analysis of Visual Scanning Patterns: A Developmental Study on Green and Normal Images," Scientific Reports, vol. 10, no. 1, pp. 1-11, 2020. [Online]. Available: http://dx.doi.org/10.1038/s41598-020-63951-3

[42] D. Hallac, S. Vare, S. Boyd, and J. Leskovec, "Toeplitz inverse covariance-based clustering of multivariate time series data," in Proceedings of the 23rd ACM SIGKDD International Conference on Knowledge Discovery and Data Mining, ser. KDD '17. New York, NY, USA: Association for Computing Machinery, 2017, p. 215-223. [Online]. Available: https://doi.org/10.1145/3097983.3098060

[43] U. Brandes, "A faster algorithm for betweenness centrality," Journal of mathematical sociology, vol. 25, no. 2, pp. 163-177, 2001. 